\title{
Cancer Genetics Services in Northern Ireland
}

\author{
P.J. Morrison and N.C. Nevin ${ }^{\#}$ \\ Regional Medical Genetics Centre, Belfast \\ City Hospital Trust, Belfast, BT9 7AB, UK
}

\section{CANCER EPIDEMIOLOGY}

Northern Ireland, like Wales and Scotland, is part of the United Kingdom. The total population in 1997 was $1,577,835$. The health care structure is similar to the rest of the UK and Northern Ireland constitutes a complete health care region within the UK. It is divided into four area health and social services boards, each providing health and social care and responsible to the Northern Ireland Department of Health. In 1993-1995, the annual average of new diagnosed cancer patients was 8513. In 1995, 2953 cancers were diagnosed in men, and the corresponding figure for women was 3162. Deaths from cancer in 1995 were 1846 and 1605 for men and women. The estimated annual incidence of specific cancers occurring within defined populations in Northern Ireland in 1997 is shown in Table 1.

\section{THE HEALTH CARE SYSTEM IN NORTHERN IRELAND}

The Northern Ireland Health Service (NHS) is available to all citizens and is government funded. Although a small number of patients have additional private healthcare plans, the NHS is the major provider of free health care services. The service is available through general practitioners that provide primary care to a network of district and regional hospitals. The

\footnotetext{
\# Correspondence: Prof. N.C. Nevin, Regional Medical Genetics Centre, Belfast City Hospital Trust, Belfast, BT9 7AB, UK, Tel.: +44 1232329241 Ext. 2764l2873, Fax: +44 1232236911
}

NHS is funded through an individual system of general tax contributions.

\section{Provision of cancer services}

Cancer genetics services in Northern Ireland, as in the rest of the UK, are modelled on the "Calman" system [1,2]. This is arranged in a three level provider service as follows:

Level 1 Primary Care: This level is provided by general practitioners (GP) with access to local routine consultation and screening for low risk patients. GP's act as gatekeepers for access and referral to specialist hospital practitioners for further screening and investigation.

Level 2 Cancer Unit: The cancer unit is organized at the district hospital level and works closely with general practitioners and other service providers in providing care for intermediate risk patients in their own local area. There are limited general oncology and surgery facilities at such hospitals. Six major hospitals provide equitable access within the four health board areas. It is planned that cancer genetic clinics will be held in these hospitals for patients and families of intermediate risk of breast and bowel cancer and some rare cancer syndromes. At present, cancer genetics services in the cancer units are provided as part of the general genetics service.

Level 3 Regional Cancer Centre: This is a specialized cancer centre serving the whole of Northern Ireland. It is located at the Belfast City Hospital, (also the base for the regional medical genetics service) and provides;

- $\quad$ specialized surgery,

- diagnostic facilities

- intensive care

- radiotherapy

- chemotherapy

- expertise in rare cancers

- medical and psychological support services 
- $\quad$ academic research and clinical trials centre.

The central cancer genetics clinics and regular high risk clinics for breast, bowel and other rare cancer syndromes are held in the Belfast City Hospital.

The Calman levels 2 and level 3 provide integrated teams of specialists, including medical oncologists, clinical geneticists, gynaecological oncologists, surgeons and psychologists. Cancer genetics clinics provide counselling, usually at a clinic with a geneticist alone but facilities for prompt referral to other cancer screening services, and related professionals. Molecular diagnostic tests are available for BRCA1, BRCA2, FAP, HNPCC, MEN 2A, MEN 2B, FMTC, and cytogenetic tests for leukaemias.

\section{THE NORTHERN IRELAND GENETIC SERVICE}

Current genetics services in the United Kingdom are organized on a regional basis with staffing ratio of 1 consultant geneticist per 500,000 population [3]. In Northern Ireland the service was established in 1967 by Professor NC Nevin. It is based in Belfast City Hospital with responsibility for providing regional genetic counselling and diagnostic clinics and laboratory services in all areas of Northern Ireland. The Cancer genetics services started in 1989, initially from a research interest in multiple endocrine neoplasia, and other rare familial cancers. Clinical research and services have expanded, and since 1995, direct mutation testing was introduced for MEN types 2A, 2B and FMTC (familial medullary thyroid cancer). In 1996, mutational analysis for familial adenomatous polyposis cancer was introduced, and subsequently for BRCA1 and BRCA 2 and bowel cancers (HNPCC). The funding for the molecular tests for cancer is from research grants. Since 1999, the regional molecular genetics laboratory, part of the Northern Ireland Regional Genetics Service, based in Belfast City Hospital offers a range of service tests, including FAP,

Table 1

Estimated incidence of specific cancers within populations served by cancer units and cancer centre

\begin{tabular}{lcc}
\hline Cancer Type & Number of cases per year per 260,000 population & Number of cases per year per 530,000 population \\
\hline Breast & 110 & 227 \\
Bladder & 79 & 163 \\
Colon & 75 & 155 \\
Kidney & 19 & 39 \\
Lung & 183 & 377 \\
Prostate & 64 & 131 \\
Rectal & 23 & 48 \\
\hline
\end{tabular}

Table 2

Cancer referrals to the Northern Ireland regional genetics service for 1997

\begin{tabular}{lc}
\hline Type of cancer & Number of referrals \\
\hline Breast & $86(60 \%)$ \\
Breast and Ovarian & $20(15 \%)$ \\
HNPCC & $15(10 \%)$ \\
Multiple endocrine (MEN 1, 2A, 2B, FMTC) & $6(5 \%)$ \\
FAP & $5(1 \%)$ \\
Li-Fraumeni & $3(1 \%)$ \\
Others & $6(5 \%)^{*}$ \\
Total & 141 \\
$*$ Includes VHL RB, NF2, WT, Ataxia telangiectasia, Cowden syndrome, juvenile & \\
polyposis, Peutz-Jeghers syndrome and other rare dominant cancer syndromes.
\end{tabular}


BRCA1 and BRCA2, MEN 2A, MEN 2B and FMTC, with supra-regional facility for retinoblastoma, Wilms tumours, Von HippelLindau disease, Li-Fraumeni syndrome, and neurofibromatosis type 2. BRCA1 and BRCA2 mutational screening is by PTT, with direct mutation screening for common mutations in FAP and MEN. Current research interests are in HNPCC, BRCA1 and 2 gene testing, and clinical and molecular aspects of multiple endocrine neoplasia.

The total referrals to the cancer genetic clinics for 1997 was 141 families. The estimated cancer referral rate for 1999 is $20-30$ cases per month. There has been steady growth in the proportion of cancer genetic referrals as in other regions of the UK, over the last 5 years.

\section{Communication of results}

Results are discussed with the patient and family by the clinical geneticist and a written report is provided to the patient and appropriate medical personnel. Clinical surveillance, according to national guidelines, is arranged for patients with a high risk and for mutation carriers for bowel and breast cancer.

\section{Staffing}

There is 1 full time genetic nurse who undertakes pre-clinic visits at which she obtains a full family history, clinical details and obtains pathological confirmation (0.3 "Whole Time Equivalent" WTE cancer genetics). A Consultant and Senior registrar contribute 0.5 WTE each to the cancer genetics clinics. There are proposals for a consultant clinical geneticist with a special interest in cancer genetics to cope with increasing demand for this service, with appropriate secretarial, data manager and junior clinical staff support. The current funding of the cancer genetics clinics is from research/charity sources and ad hoc NHS funding. It is proposed that additional service cytogenetic and molecular genetic laboratory staff will be funded through the NHS.

\section{Audit of services}

All cancer genetic clinics are subject to continuing audit and evaluation of service.

\section{Criteria for testing}

1. Amsterdam criteria for HNPCC:

- 3 cases of colorectal cancer in the family

- 1 is a $1^{\text {st }}$ degree relative of the other two

- 1 under 50 years

- $\quad$ at least two generations affected

Modified criteria: one of the cases could be endometrial cancer, or other recognised cancers in Lynch II syndrome including; gastric cancer, uroepithelial cancer, multiple basal cell carcinomas or ovarian cancer.

\section{Breast cancer referral criteria:}

- 3 or more close relatives (same side of family) with breast or ovarian cancer

- 2 close relatives (same side of family) with breast cancer under the age of 50

- 1 first degree relative with breast cancer under 40

- $\quad$ any male breast cancer

- $1^{\text {st }}$ degree relative with bilateral breast cancer or breast cancer and ovarian cancer

- history of breast and other cancers in three or more close relatives (same side of family) affected at younger than average age

- history of breast cancer and childhood cancer

3. Ovarian cancer referral criteria:

- 2 or more close relatives (same side of family) with ovarian cancer at any age

- 1 ovarian cancer at any age and 1 breast cancer under 50 or two or more breast cancers at any age

- family history fitting a Lynch II pattern with ovarian cancer

\section{Other cancers:}

Medullary thyroid cancer (MEN 2A, 2B, etc.): Any case with medullary C-cell involvement, or history or suspicion of parathyroid or adrenal involvement. 
Neurofibromatosis: usually no DNA testing for Type I as mainly clinical diagnosis, DNA testing for suitable type II case dependent on opinion of clinical geneticist, neurosurgeon or ENT surgeon.

Other potentially autosomal dominant childhood cancers - Retinoblastoma, Wilm's tumour, Ataxia telangiectasia, aniridia.

Any cancer family history with significant patient concern

\section{Breast cancer risk:}

The population risk of breast cancer in Northern Ireland is 1 in 12 . Medium risk defined as 1 in 6 , to 1 in 4 . High risk defined as $\geq 1$ in 4 .

\section{References}

[1] Department of Health. Genetics and cancer services. Report of a working group for the chief medical officer. London: Department of Health. 1998.

[2] Department of Health and Social Services. Cancer services, investing for the future. Report of the cancer working group. Belfast: Department of Health and Social Services for Northern Ireland, 1996.

[3] Royal College of Physicians. Commissioning clinical genetic services. A working Party report. London Royal College of Physicians. 1999. 


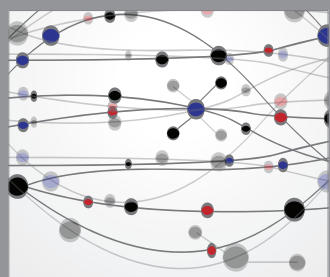

The Scientific World Journal
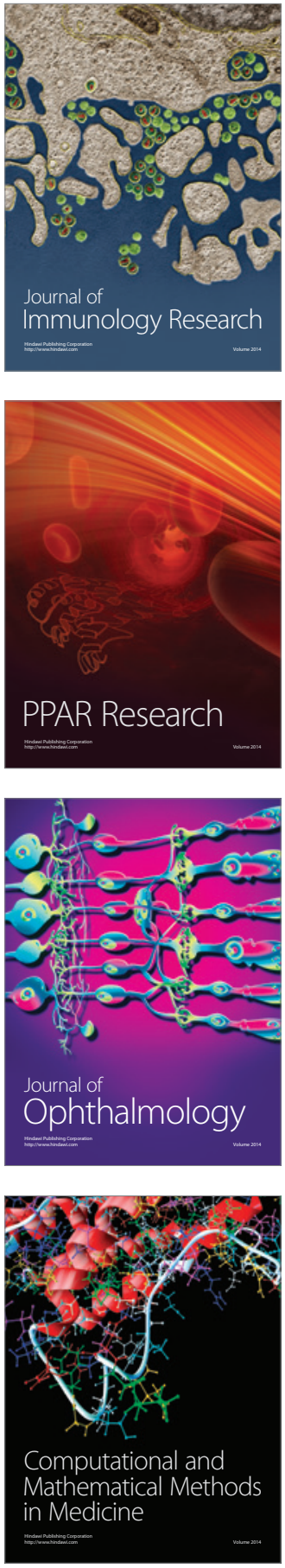

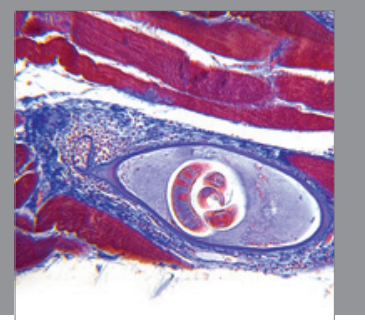

Gastroenterology

Research and Practice
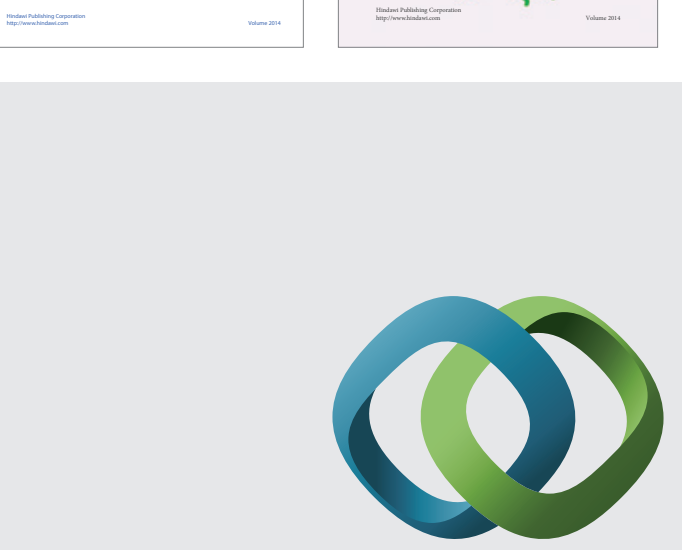

\section{Hindawi}

Submit your manuscripts at

http://www.hindawi.com
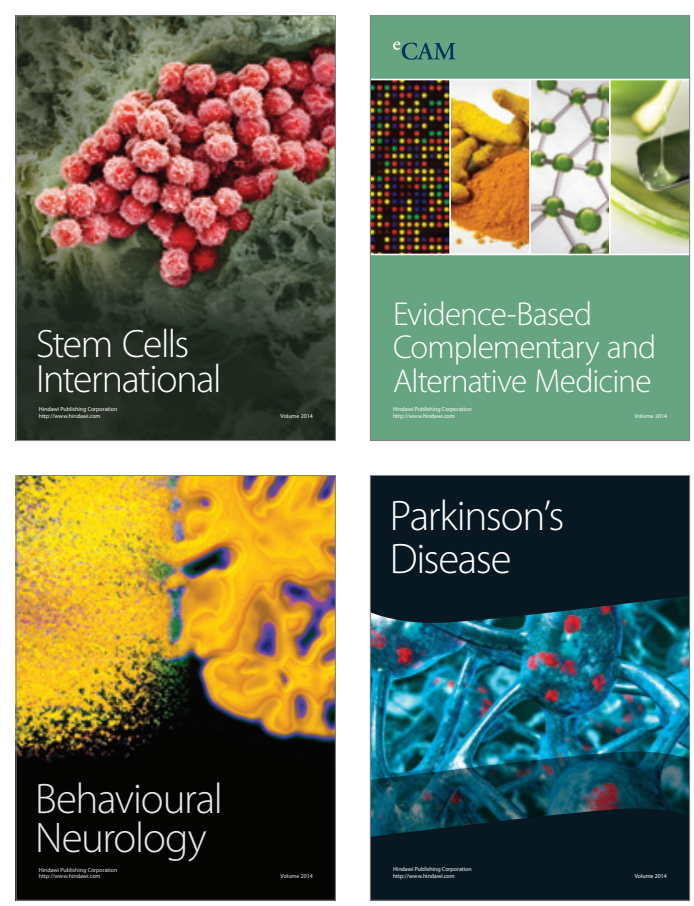

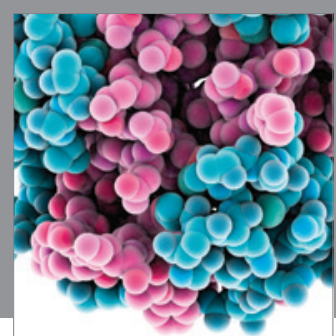

Journal of
Diabetes Research

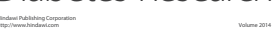

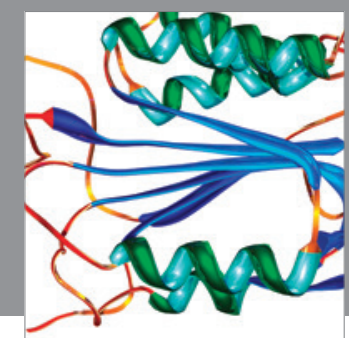

Disease Markers
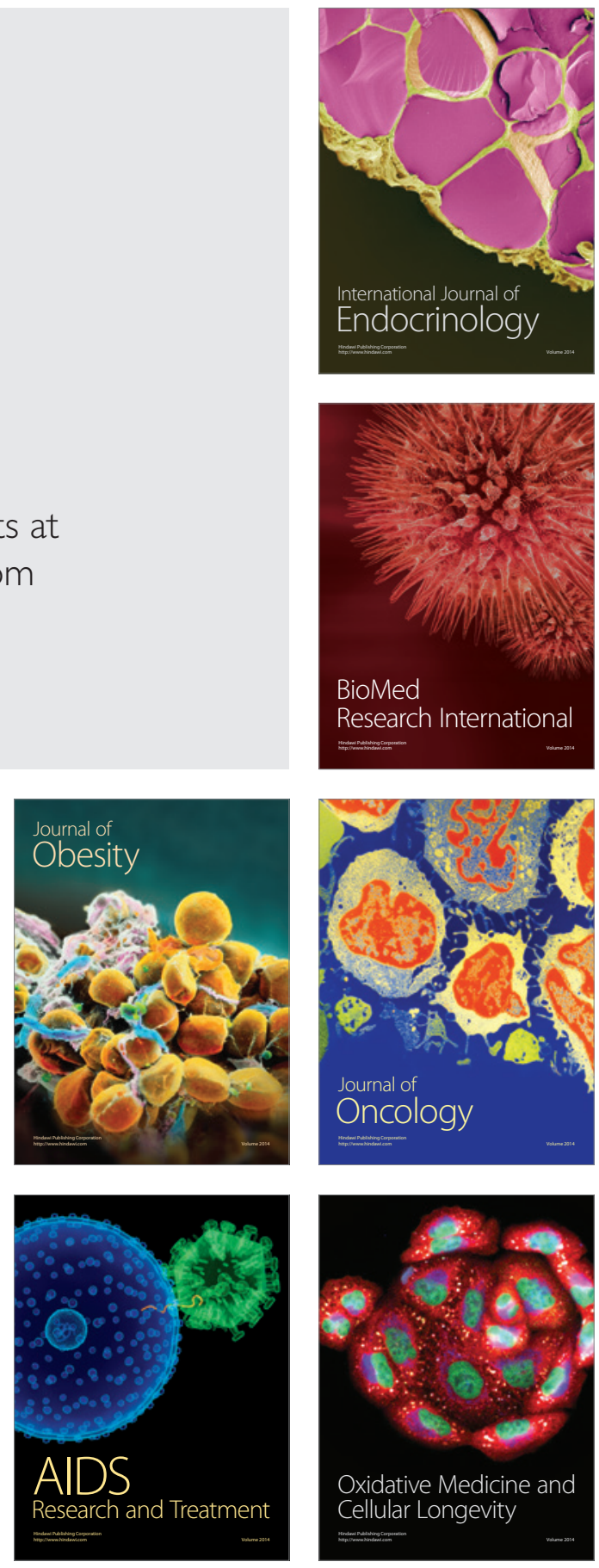